DFTT $75 / 96$

GEF-Th-19/96

ROM2F-96/63

December 1996

\title{
Exploring the supersymmetric parameter space by direct search for WIMPs
}

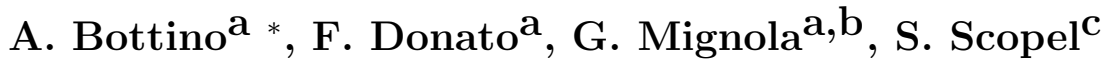 \\ ${ }^{a}$ Dipartimento di Fisica Teorica, Università di Torino and \\ INFN, Sezione di Torino, Via P. Giuria 1, 10125 Turin, Italy \\ ${ }^{b}$ LAPP - Annecy Le Vieux, Chemin de Bellevue BP 110-F-74941 Annecy-Le-Vieux, France \\ ${ }^{c}$ Dipartimento di Fisica, Università di Genova and \\ INFN, Sezione di Genova, Via Dodecaneso 33, 16146 Genoa, Italy

\section{P. Belli ${ }^{\mathrm{d}}$, A. Incicchitti ${ }^{\mathrm{e}}$} \\ d Dipartimento di Fisica, Università di Roma "Tor Vergata" \\ and INFN, sez Roma II, I-00173 Rome, Italy \\ e Dipartimento di Fisica, Università di Roma "La Sapienza" \\ and INFN, sez. Roma, I-00165 Rome, Italy
}

\begin{abstract}
It is shown that the new data of the DAMA/NaI detector, combined with recent calculations of nuclear structure functions, provide the most stringent upper bounds on the WIMP-nucleon cross-section both for spin-dependent and for scalar interactions. It is proved that, for the first time, data from an experiment of direct search for WIMPs allow the exploration of a region of the supersymmetric parameter space not yet fully investigated at accelerators.
\end{abstract}

\footnotetext{
*E-mail: bottino@to.infn.it,donato@to.infn.it, mignola@to.infn.it,scopel@ge.infn.it, belli@roma2.infn.it, incicchitti@vaxrom.roma1.infn.it
} 


\section{INTRODUCTION}

An experiment for direct detection of Weakly Interacting Massive Particle (WIMP), which employs a large-mass, low-background $\mathrm{NaI}(\mathrm{Tl})$ detector at the Gran Sasso Laboratory [1], has recently allowed a substantial improvement of the upper limit on the WIMP-nucleon elastic scattering cross-section. A high exposure of $4123 \mathrm{Kg}$ day, a strong reduction of the residual internal contaminants and a statistical pulse shape discrimination have made this result possible.

In this letter we examine the impact that this new measurement has in terms of relic neutralinos, and more generally in terms of an exploration of the supersymmetric parameter space. First, in Sect.2 we discuss how upper bounds on (scalar and spin-dependent) WIMP-nucleon cross-sections are extracted from the experimental counting rates. Whereas the procedure is straightforward for the scalar case, for the spin-dependent case appropriate specifications on the interaction mechanism and realistic nuclear-physics calculations are needed. We show how the new data from the DAMA/NaI-detector provide the most stringent upper bounds both to scalar and to spin-dependent WIMP-nucleon cross-sections (except for a very narrow range of the WIMP mass around $15 \mathrm{GeV}$ ). This remarkable result derives from the significant improvement in sentivity in the experiment of Ref. [1] and, for the spin-dependent part, also from new theoretical calculations of nuclear effects [2,3]. Then, in Sect.3 we proceed to a theoretical analysis of the implications that the new experimental data have for relic neutralinos, and we show that, for the first time, data from an experiment of direct search for WIMPs allow the exploration of some regions of the supersymmetric parameter space, whose investigation at accelerators is still under way.

\section{RATES FOR DIRECT DETECTION}

The experimental activity in the direct search for relic particles has been very intense in recent years [4 17, with quite significant improvements in various technical aspects that have drawn the experimental sensitivity nearer and nearer to the one required to match

the predictions of realistic theoretical models. It is well known that the only safe way of discriminating a real signal for WIMPs from the background is to measure a modulation effect [18]. Some preliminary measurements [12,16, 17] have already been performed, but the search for modulation will have to be pursued with increased sensitivity in order to reach the level required by a number of specific dark matter candidates (such as the neutralino). Since the current counting rates are still expected to be dominated by background effects, only upper bounds on WIMP-nucleus cross-sections can be derived at present. Some of the considerations presented in this section apply to a generic WIMP, however, since we will concentrate on the neutralino $(\chi)$ later on, when we compare experimental bounds with theoretical evaluations, we start from the very beginning with a formalism which applies directly to this favorite particle candidate for cold dark matter. 
The neutralino is defined as the linear superposition

$$
\chi=N_{1} \tilde{\gamma}+N_{2} \tilde{Z}+N_{3} \tilde{H}_{1}^{\circ}+N_{4} \tilde{H}_{2}^{\circ},
$$

of lowest mass. Here $\tilde{\gamma}, \tilde{Z}$ are the photino and zino states and $\tilde{H}_{1}^{\circ}, \tilde{H}_{2}^{\circ}$ are the higgsino fields, supersymmetric partners of the Higgs fields $H_{1}^{\circ}, H_{2}^{\circ}$. The theoretical framework used in this paper for the supersymmetric model will be presented in Sect.3.

We write the differential event rate for elastic neutralino-nucleus scattering as

$$
\frac{d R}{d E_{R}}=N_{T} \frac{\rho_{\chi}}{m_{\chi}} \int_{v_{\min }\left(E_{R}\right)}^{v_{\max }} d v f(v) v \frac{d \sigma}{d E_{R}}\left(v, E_{R}\right) .
$$

$N_{T}$ is the number density of the detector nuclei, $\rho_{\chi} / m_{\chi}$ is the local (solar neighbourhood) number density of neutralinos, $f(v)$ is the velocity distribution of neutralinos in the Galaxy (assumed to be Maxwellian in the Galactic rest frame) evaluated in the Earth's rest frame, $d \sigma / d E_{R}$ is the elastic differential neutralino-nucleus cross section, $E_{R}=m_{r e d}^{2} v^{2}\left(1-\cos \theta^{*}\right) / m_{N}$ is the recoil energy $\left(\theta^{*}\right.$ is the scattering angle in the neutralinonucleus center of mass frame), $m_{\text {red }}$ is the neutralino-nucleus reduced mass and $m_{N}$ is the nuclear mass. Eq.(2) is written for a monoatomic material; its generalization to more complex materials is straightforward.

\section{A. Astrophysical and cosmological parameters}

Many astrophysical quantities, affected by large uncertainties, enter in the evaluation of the differential event rate. This is the case for the neutralino r.m.s. velocity and for its escape velocity, whose typical values are: $v_{r . m . s .}=270 \pm 24 \mathrm{~km} \cdot \mathrm{s}^{-1}$ [19], $v_{e s c}=650 \pm 200 \mathrm{~km} \cdot \mathrm{s}^{-1}$ [20] and for the velocity of the Sun around the galactic centre $\left(v_{\odot}=232 \pm 20 \mathrm{~km} \cdot \mathrm{s}^{-1}\right.$ [19]).

Also a large uncertainty concerns the value of the local dark matter density $\rho_{l}$. A recent determination of $\rho_{l}$, based on a flattened dark matter distribution and microlensing data, gives the range $\rho_{l}=0.51_{-0.17}^{+0.21} \mathrm{GeV} \cdot \mathrm{cm}^{-3}$ 21]. In particular the central value turns out to be significantly larger than the one previously determined, for instance, in Ref. [22]: $\rho_{l}=0.3 \pm 0.1 \mathrm{GeV} \cdot \mathrm{cm}^{-3}$. Furthermore, for any specific value for the local density of the total dark matter $\rho_{l}$, one has to assign a value to the neutralino local density $\rho_{\chi}$. To determine the value of $\rho_{\chi}$ to be used in Eq.(2), we adopt the following rescaling recipe [23]: for each point of the parameter space, we take into account the relevant value of the cosmological neutralino relic density. When $\Omega_{\chi} h^{2}$ is larger than a minimal $\left(\Omega h^{2}\right)_{\min }$, compatible with observational data and with large-scale structure calculations, we simply put $\rho_{\chi}=\rho_{l}$. When $\Omega_{\chi} h^{2}$ turns out to be less than $\left(\Omega h^{2}\right)_{\text {min }}$, and then the neutralino may 
TABLE I. Values of the astrophysical and cosmological parameters relevant to direct detection rates. $V_{\text {r.m.s. }}$ denotes the root mean square velocity of the neutralino Maxwellian velocity distribution in the halo; $V_{e s c}$ is the neutralino escape velocity and $V_{\odot}$ is the velocity of the Sun around the galactic centre; $\rho_{l o c}$ denotes the local dark matter density and $\left(\Omega h^{2}\right)_{\min }$ the minimal value of $\Omega h^{2}$. The values of set I are the median values of the various parameters, the values of set II are the extreme values of the parameters which, within the physical ranges, provide the lowest estimates of the direct rates (once the supersymmetric parameters are fixed).

\begin{tabular}{|c|r|r|}
\hline \hline & Set I & Set II \\
\hline$V_{r . m . s}\left(\mathrm{~km} \cdot \mathrm{s}^{-1}\right)$ & 270 & 245 \\
\hline$V_{\text {esc }}\left(\mathrm{km} \cdot \mathrm{s}^{-1}\right)$ & 650 & 450 \\
\hline$V_{\odot}\left(\mathrm{km} \cdot \mathrm{s}^{-1}\right)$ & 232 & 212 \\
\hline$\rho_{l o c}\left(\mathrm{GeV} \cdot \mathrm{cm}^{-3}\right)$ & 0.5 & 0.2 \\
\hline$\left(\Omega h^{2}\right)_{\min }$ & 0.03 & 0.3 \\
\hline \hline
\end{tabular}

only provide a fractional contribution $\Omega_{\chi} h^{2} /\left(\Omega h^{2}\right)_{\text {min }} \equiv \xi$ to $\Omega h^{2}$, we take $\rho_{\chi}=\rho_{l} \xi$. The value to be assigned to $\left(\Omega h^{2}\right)_{\min }$ is somewhat arbitrary, in the range $0.03 \lesssim\left(\Omega h^{2}\right)_{\min } \lesssim 0.3$.

In Table I we give the two sets of astrophysical and cosmological parameters that we use in this letter. Set I corresponds essentially to the central values for the various parameters, whereas set II corresponds to those values of the parameters, which, within the relevant allowed ranges, provide the lowest detection rates (once the supersymmetric variables are fixed).

\section{B. Cross-sections}

The differential cross-section $\frac{d \sigma}{d E_{R}}\left(v, E_{R}\right)$ in Eq.(2) may conveniently be splitted into a coherent part and a spin-dependent one [24]

$$
\frac{d \sigma}{d E_{R}}=\left(\frac{d \sigma}{d E_{R}}\right)_{C}+\left(\frac{d \sigma}{d E_{R}}\right)_{S D} .
$$

When the WIMP is the neutralino, the coherent cross-section, $\left(\frac{d \sigma}{d E_{R}}\right)_{C}$, originates from Higgs-exchange [25] and squark-exchange [26] in the neutralino-nucleus scattering; the spindependent cross-section $\left(\frac{d \sigma}{d E_{R}}\right)_{S D}$ is due to Z-exchange and to squark-exchange [26]. The links among supersymmetric and nuclear degrees of freedom have quite different features in the coherent and in the spin-dependent cross-sections. Thus, let us consider the two cases separately. 


\section{Coherent $\chi$-nucleus cross-section}

In the case of the coherent cross-section, supersymmetric and nuclear degrees of freedom decouple, thus $\left(\frac{d \sigma}{d E_{R}}\right)_{C}$ may be written as

$$
\left(\frac{d \sigma}{d E_{R}}\right)_{C}=\frac{\sigma_{C}^{0}}{E_{R}^{\max }} F^{2}(q)
$$

where $\sigma_{C}^{0}$ is the total coherent cross-section, conventionally integrated from zero up to $E_{R}^{\max }$ $=2 m_{r e d}^{2} v^{2} / m_{N}, q^{2} \equiv \vec{q}^{2}=2 m_{N} E_{R}$ is the squared three-momentum transfer, and $F_{C}(q)$ is a nuclear form factor defined as the Fourier transform of the nuclear matter distribution. Except for very light nuclei, which have a form factor $F_{C}$ of exponential or gaussian type, a universal parametrization for $F_{C}(q)$ is provided by [27]

$$
F(q)=3 \frac{j_{1}\left(q r_{0}\right)}{q r_{0}} e^{-\frac{1}{2} s^{2} q^{2}}
$$

where $j_{1}\left(q r_{0}\right)$ is the spherical Bessel function of index $1, s \simeq 1 \mathrm{fm}$ is the thickness parameter for the the nuclear surface, $r_{0}=\left(R^{2}-5 s^{2}\right)^{1 / 2}$ and $R=1.2 A^{1 / 3} \mathrm{fm}$ ( $A$ is the atomic number).

The cross-section $\sigma_{C}^{0}$ may be written as

$$
\sigma_{C}^{0}=\frac{8 G_{F}^{2}}{\pi} M_{Z}^{2} \zeta^{2} m_{r e d}^{2} A^{2}
$$

where the quantity $\zeta$ depends on the $\chi$-quark couplings mediated by Higgs particles and squarks [25,26]. The full expression for $\zeta$ together with the values used here for the relevant parameters are reported in Ref. [28]. It is worth recalling that the simple dependence of $\sigma_{C}$ on $A^{2}$ is due to the equality (within a very good accuracy) of the $\chi$-neutron and the $\chi$-proton couplings.

From the factorization property inherent in the structure of Eq.(6) it follows that $\sigma_{C}^{0}$ may be immediately converted into an equivalent $\chi-$ nucleon scalar cross section

$$
\sigma_{\text {scalar }}^{(\text {nucleon })}=\frac{1+m_{\chi}^{2} / m_{N}^{2}}{1+m_{\chi}^{2} / m_{p}^{2}} \frac{\sigma_{C}^{0}}{A^{2}}
$$

where $m_{p}$ denotes the proton mass. Eqs.(4-1) enable a straightforward conversion of any upper limit on $\frac{d \sigma}{d E_{R}}$ into an upper bound on $\sigma_{\text {scalar }}^{\text {(nucleon })}$. This procedure allows a direct comparison among the results of various experiments. It is obvious that this method applies also to any other WIMP which couples with equal strength to neutrons and to protons. 
In Fig. 1 we present a summary of the current upper bounds, obtained by the method previously described. All experimental data have been treated with the same algorithm and with the same set of astrophysical parameters (set I above; $\left(\Omega h^{2}\right)_{\min }$ is not relevant here). We notice that the upper limit derived from the DAMA/NaI experiment provides the most stringent upper bound on the scalar neutralino-nucleon cross-section with an improvement of a factor $3-5$ over the limits presently placed by Ge-detectors. The only exception is represented by a very narrow range in the neutralino mass around $m_{\chi} \simeq 15 \mathrm{GeV}$. However, except for peculiar values of the other supersymmetric parameters, this range in $m_{\chi}$ is below the current lower bound on the neutralino mass [29, 30].

\section{Spin-dependent $\chi$-nucleus cross-section}

Let us turn now to the cross-section due to spin-dependent interactions. Using standard notations, we write the differential cross-section $\frac{d \sigma}{d E_{R}}$ for neutralino-nucleus scattering as

$$
\left(\frac{d \sigma}{d E_{R}}\right)_{S D}=\frac{16 G_{F}^{2}}{v^{2}} m_{N} \frac{S(q)}{2 J+1} .
$$

where $J$ is the nuclear spin and $S(q)$ is given by:

$$
S(q)=a_{0}^{2} S_{00}(q)+a_{1}^{2} S_{11}(q)+a_{0} a_{1} S_{01}(q)
$$

The functions $S_{i j},(i, j=0,1)$ are rather complicated functions which depend on the nuclear spin structure and on the neutralino composition; their definitions may be found in Ref. [31. The coefficients $a_{0}$ and $a_{1}$ are the isoscalar and isovector projections of the neutralinoneutron and neutralino-proton couplings $a_{n}, a_{p}$, respectively, i.e. $a_{0}=a_{p}+a_{n}, a_{1}=a_{p}-a_{n}$. The full expressions for $a_{n}, a_{p}$ are reported, for instance, in Refs. [28, 32]. In the case of the $\chi$-nucleon interaction mediated by Z-exchange one has

$$
a_{p, n}=-\frac{1}{2}\left(N_{3}^{2}-N_{4}^{2}\right)\left(\sum_{q=u, d, s} T_{3 q} \Delta q\right)_{p, n}
$$

where $T_{3 q}$ denotes the third component of the quark weak isospin, $\Delta q$ is the fractional spin carried by the quark q in the appropriate nucleon and $N_{3}, N_{4}$ are the Higgsino coefficients in the expression for $\chi$ (see Eq.(便)).

The cross-section of Eq.(8) may be conveniently rewritten as [33] 


$$
\left(\frac{d \sigma}{d E_{R}}\right)_{S D}=\frac{16 G_{F}^{2}}{v^{2} \pi} m_{N} \Lambda^{2} J(J+1) \frac{S(q)}{S(0)}
$$

where the static value of $S(0)$ has been expressed as

$$
S(0)=\frac{2 J+1}{\pi} \Lambda^{2} J(J+1)
$$

with

$$
\Lambda=\frac{a_{p}<\vec{S}_{p}>+a_{n}<\vec{S}_{n}>}{J}
$$

$<\vec{S}_{p}>$ and $\left\langle\vec{S}_{n}>\right.$ are the contributions of the total proton and neutron spins in the nucleus. Another commonly used notation is $\lambda_{p(n)}=\Lambda / a_{p(n)}$.

It is important to recall that both $\Lambda$ and $S(q) / S(0)$ depend on nuclear properties as well as on the neutralino composition. Thus, in spin-dependent interactions nuclear degrees of freedom and supersymmetric degrees of freedom do not decouple in the most general case. This feature does not allow an extraction of a universal (i.e. independent of the neutralino composition) spin-dependent $\chi$-nucleon cross-section in the same way as was done in the coherent case.

An exception is provided by spin-dependent interactions mediated by Z-exchange only. This property is explicitely exhibited by the expressions of Eq.(10) for $a_{p}, a_{n}$, which imply

$$
\left[a_{n} / a_{p}\right]_{Z}=\left(\sum_{q} T_{3 q} \Delta q\right)_{n} /\left(\sum_{q} T_{3 q} \Delta q\right)_{p}
$$

In order to compare experimental data due to various detectors in a way similar to the one previously employed for the coherent interactions, in the rest of this section we expand the formalism in the case of a pure Z-exchange contribution to the spin-dependent scattering. Under this assumption, the spin-dependent cross-section may be cast into the form

$$
\left(\frac{d \sigma}{d E_{R}}\right)_{S D}=\frac{4 G_{F}^{2}}{\pi v^{2}} m_{N}\left(N_{3}^{2}-N_{4}^{2}\right)^{2}\left(\sum_{q} T_{3 q} \Delta q\right)_{p}^{2} \lambda_{p, Z}^{2} J(J+1) F_{S D}^{2}(q)
$$

where $\lambda_{p, Z}$ is defined as 


$$
\lambda_{p, Z} \equiv\left[\frac{\Lambda}{a_{p}}\right]_{a_{n} / a_{p}=\left[a_{n} / a_{p}\right]_{Z}}=\frac{1}{J}\left[<\vec{S}_{p}>+\frac{a_{n}}{a_{p}}<\vec{S}_{n}>\right]_{a_{n} / a_{p}=\left[a_{n} / a_{p}\right]_{Z}}
$$

and

$$
F_{S D}^{2}(q) \equiv\left[\frac{S(q)}{S(0)}\right]_{a_{n} / a_{p}=\left[a_{n} / a_{p}\right] Z}
$$

Thus we may further define a total point-like spin-dependent cross-section (conventionally integrated from zero up to $\left.\left(E_{R}\right)_{\max }\right)$ as

$$
\begin{aligned}
\sigma_{S D}^{0} & =\left(E_{R}\right)_{\max }\left(\frac{d \sigma}{d E_{R}}\right)_{S D} \frac{1}{F_{S D}^{2}(q)} \\
& =\frac{8 G_{F}^{2}}{\pi} m_{r e d}^{2}\left(N_{3}^{2}-N_{4}^{2}\right)^{2}\left(\sum_{q} T_{3 q} \Delta q\right)_{p}^{2} \lambda_{p, Z}^{2} J(J+1),
\end{aligned}
$$

in terms of which we may obtain an equivalent $\chi$-proton spin-dependent cross-section

$$
\sigma_{S D}^{(\text {proton })}=\frac{1+m_{\chi}^{2} / m_{N}^{2}}{1+m_{\chi}^{2} / m_{p}^{2}} \frac{3}{4} \frac{\sigma_{S D}^{0}}{\lambda_{p, Z}^{2} J(J+1)}
$$

Therefore, under the assumption of a pure (or dominant) Z-exchange, Eqs.(15-19) allow the conversion of any experimental upper limit on $\frac{d \sigma}{d E_{R}}$ into an upper bound on $\sigma_{S D}^{(\text {proton })}$, in much the same way as for the coherent case. However, still an important difference between the two cases concerns the features of the nuclear properties. Whereas in the coherent case these are simply taken into account by the form factor $F_{C}$ of the universal analytic form of Eq.(5), in the spin-dependent one the nuclear properties have to be calculated for each nucleus with an appropriate nuclear model. In our analysis of the spin-dependent crosssections we have employed the most recent nuclear-physics calculations for the structure functions $S_{i j}(q)$ for the nuclei of interest. From the previous formulae it is clear that from these $S_{i j}$ 's both the static value $\lambda$ and the form factor $F_{S D}$ are readily derived.

The nuclei with non-vanishing spin, relevant for our analysis, are ${ }^{23} \mathrm{Na},{ }^{73} \mathrm{Ge},{ }^{127} \mathrm{I},{ }^{129} \mathrm{Xe}$. For these nuclei we give in Table II the values for $\lambda_{p, Z}^{2} J(J+1)$ used in our analysis [2, [3] 36], compared with some of the previous determinations [34.355. We have set $a_{n} / a_{p}=\left[a_{n} / a_{p}\right]_{Z}$, and we have assigned to this ratio the value $\left[a_{n} / a_{p}\right]_{Z}=-0.85$, which is obtained from Eq.(10) using for the $\Delta q$ 's the estimates of Ref. [37]. 
TABLE II. Values of the nuclear spin-dependent static properties. In the first column are listed the nuclei with non-vanishing spin considered in the present paper. Their nuclear spins are given in the second column. In the third, fourth and fifth columns are listed the values of $\left\langle\vec{S}_{p}\right\rangle,\left\langle\vec{S}_{n}\right\rangle$ and $\lambda_{p, Z}^{2} J(J+1)$ derived from the following references: Ref. [3] for ${ }^{23} \mathrm{Na}$ and ${ }^{129} \mathrm{Xe}$, Ref. [36] for ${ }^{73} \mathrm{Ge}$, and Ref. [2, 3] for ${ }^{127} \mathrm{I}$. In the sixth column is given the ratio $\left(\lambda_{p(n), Z} / \lambda_{O G}\right)^{2}$ : the numerator stands for the $\lambda$ factor for proton or for neutron, according to whether protons or neutrons are the odd species in the relevant nucleus $\left(\lambda_{p, Z}\right.$ for ${ }^{23} \mathrm{Na}$ and ${ }^{127} \mathrm{I}$, and $\lambda_{n, Z}$ for ${ }^{73} \mathrm{Ge}$ and $\left.{ }^{129} \mathrm{Xe}\right)$, and its value is obtained from the Refs. [2,3, 36]; the denominator stands for previous evaluations with the odd-group approximation for ${ }^{23} \mathrm{Na},{ }^{73} \mathrm{Ge}$ and ${ }^{129} \mathrm{Xe}$ [34 and with the interacting boson fermion model for ${ }^{127} \mathrm{I}$ [35]. (B) and $(\mathrm{N})$ denote that the nuclear-physics calculations of Refs. [2, 3] were performed with the Bonn A or with the Nijmegen II nucleon-nucleon potential, respectively.

\begin{tabular}{|c|r|r|r|r|r|}
\hline \hline nucleus & $\mathrm{J}$ & $\left.<\vec{S}_{p}\right\rangle$ & $\left\langle\vec{S}_{n}\right\rangle$ & $\lambda_{p, Z}^{2} J(J+1)$ & $\lambda_{p(n), Z}^{2} / \lambda_{O G}^{2}$ \\
\hline \hline${ }^{23} \mathrm{Na}$ & $3 / 2$ & 0.248 & 0.020 & 0.089 & 2.2 \\
\hline${ }^{73} \mathrm{Ge}$ & $9 / 2$ & 0.030 & 0.378 & 0.103 & 2.2 \\
\hline${ }^{127} \mathrm{I}(B)$ & $5 / 2$ & 0.309 & 0.075 & 0.084 & 3.6 \\
\hline${ }^{127} \mathrm{I}(N)$ & $5 / 2$ & 0.354 & 0.064 & 0.126 & 5.5 \\
\hline${ }^{129} \mathrm{Xe}(B)$ & $1 / 2$ & 0.028 & 0.358 & 0.229 & 2.5 \\
\hline${ }^{129} \mathrm{Xe}(N)$ & $1 / 2$ & 0.013 & 0.300 & 0.177 & 2.0 \\
\hline \hline
\end{tabular}

It is noticeable that the new evaluations of the static properties provide larger values for $\lambda$ as compared to the previous ones. We remark that these quantities are somewhat sensitive to the type of the nucleon-nucleon interactions used in the calculations.

The form factor $F_{S D}$ for the various nuclei has been calculated using the nuclear-physics results of Refs. [2, 3],36]. The relevance of these finite-size effects depend on the range of the variable $\mathrm{qR}$ which is employed for the extraction of the upper bounds on the differential counting rates. This, in turn, depends (apart from the nuclear size) on the other experimental features: energy threshold and level of the counting rates. For ${ }^{23} \mathrm{Na}$ and ${ }^{73} \mathrm{Ge}$ finite size effects are negligeable, since the relevant $\mathrm{qR}$ values are $0.27 \leq \mathrm{qR} \leq 0.55$ for ${ }^{23} \mathrm{Na}$ and $0.75 \leq \mathrm{qR} \leq 1.4$ for ${ }^{73} \mathrm{Ge}$. On the contrary, these effects have to be properly taken into account for ${ }^{127} \mathrm{I}$ and ${ }^{129} \mathrm{Xe}$, whose $\mathrm{qR}$ ranges are: $2.2 \leq \mathrm{qR} \leq 4.4$ for ${ }^{127} \mathrm{I}$ and $1.6 \leq \mathrm{qR} \leq 3.5$ for ${ }^{129} \mathrm{Xe}$. In Fig.2 are shown the plots of the function $\left[S(q) / a_{p}^{2}\right]_{Z} \equiv\left[S(q) / a_{p}^{2}\right]_{a_{n} / a_{p}=\left[a_{n} / a_{p}\right]_{Z}}$ for these two nuclei, calculated from the structure functions $S_{i j}$ 's of Refs. [2,3] for the two different nucleon-nucleon interactions previously mentioned. Again we have set $a_{n} / a_{p}=\left[a_{n} / a_{p}\right]_{Z}=-0.85$. It is worth noticing two main features: i) the shape of $S(q)$ is not very sensitive to the form of the nucleon-nucleon interaction, except for ${ }^{129} \mathrm{Xe}$ for $\mathrm{qR} \gtrsim 4$; a region which is, however, beyond the qR-range employed in our extraction of the upper limits, ii) the function $S(q)$ for ${ }^{127} \mathrm{I}$ shows a plateau for $\mathrm{qR} \gtrsim 3$, then in a region which is important in our analysis.

When we apply the procedure previously outlined (i.e. Eqs.(15-19)) to the counting 
rates of the experiments of Refs. 11,5,9 11, 13 we finally obtain the results shown in Fig.3. For the quenching factor $\mathrm{Q}$ the following values have been used: $\mathrm{Q}=0.30$ for ${ }^{23} \mathrm{Na}$ [1, 1, 11, 1 , $\mathrm{Q}=0.25$ for ${ }^{73} \mathrm{Ge}$ [5], $\mathrm{Q}=0.09$ for ${ }^{127} \mathrm{I}$ [1, [7, 11] and $\mathrm{Q}=0.65$ for ${ }^{129} \mathrm{Xe}$ [12, 14]. From this figure it turns out that the data of the DAMA/NaI provide the most stringent upper bound also for the spin-dependent cross-section $\sigma_{S D}^{(\text {proton })}$. It is noticeable that, because of the new higher value for $\lambda$ and of the property ii) discussed above, the constraint from the I-nucleus turns out to be significantly stronger as compared to results of previous analyses.

\section{EVALUATION OF CROSS-SECTIONS IN THE MSSM}

The supersymmetric model employed in this paper is the so-called Minimal Supersymmetric extension of the Standard Model (MSSM) [38], which provides the most convenient phenomenological framework at the electroweak scale $\left(M_{Z}\right)$ and does not assume too strong, arbitrary theoretical hypotheses (for instance, universality conditions for the soft scalar masses at the Grand Unified scale $M_{G U T}$ ). Various properties (relic abundances and detection rates) of relic neutralinos have been analyzed in the MSSM by a number of authors. Some of the most recent ones are given in Refs. [39, 32,40, 42].

The MSSM is based on the same gauge group as the Standard Model and contains the supersymmetric extension of its particle content. The Higgs sector is modified as compared to that of the Standard Model, because it requires two Higgs doublets $H_{1}$ and $H_{2}$ in order to give mass both to down- and up-type quarks and to cancel anomalies. Thus the MSSM contains three neutral Higgs fields: a pseudoscalar and two scalar fields. The Higgs sector is specified at the tree level by two independent parameters: the mass of a physical Higgs field (we use the mass $m_{A}$ of the neutral pseudoscalar boson) and the ratio of the two vacuum expectation values, usually defined as $\tan \beta=v_{2} / v_{1} \equiv<H_{2}>/<H_{1}>$. Other important ingredients of the model are, apart from the Yang-Mills Lagrangian, the superpotential, which contains all the Yukawa interactions and the Higgs-mixing term $\mu H_{1} H_{2}$, and the soft-breaking Lagrangian, containing the trilinear and bilinear breaking parameters and the soft gaugino and scalar masses.

The theoretical model contains an exceedingly large number of parameters, unless some assumptions are introduced. It is customary to introduce the following conditions (at the $M_{Z}$ scale): i) all trilinear parameters are zero except those of the third family, which however have a common value $\mathrm{A}$, ii) all squarks are taken as degenerate: $m_{\tilde{q}_{i}} \equiv m_{0}$, except for $m_{\tilde{t}}$, iii) all sleptons are taken as degenerate with $m_{\tilde{l}_{i}} \equiv m_{0}$, iv) the gaugino masses are assumed to unify at $M_{G U T}$; thus in particular $M_{1}=(5 / 3) \tan ^{2} \theta_{W} M_{2}$.

After these conditions are applied, the number of independent parameters reduces to seven. We choose them to be: $M_{2}, \mu, \tan \beta, m_{A}, m_{0}, m_{\tilde{t}}, \mathrm{~A}$. In terms of these parameters, the supersymmetric space is further constrainted by the following requirements: 1) all experimental bounds on Higgs, neutralino, chargino and sfermion masses are satisfied (taking 
into account also the new data from LEP2 [29,43, 44]), 2) the neutralino is the Lightest Supersymmetric Particle (LSP) (i.e., regions where gluino or squarks or sleptons are LSP are excluded), 3) constraints due to the $b \rightarrow s+\gamma$ process [45 are satisfied, 4) the neutralino relic abundance does not exceed the cosmological bound, i.e. $\Omega_{\chi} h^{2} \leq 1$.

The results presented in this paper for the evaluations of neutralino-nucleon cross sections within MSSM have been obtained by varying the independent parameters of the model in the following ranges: $10 \mathrm{GeV} \leq M_{2} \leq 500 \mathrm{GeV}, 10 \mathrm{GeV} \leq|\mu| \leq 500 \mathrm{GeV}, 60 \mathrm{GeV} \leq$ $m_{A} \leq 500 \mathrm{GeV}, 100 \mathrm{GeV} \leq m_{0} \leq 500 \mathrm{GeV}, 100 \mathrm{GeV} \leq m_{\tilde{t}} \leq 500 \mathrm{GeV},-3 \leq \mathrm{A} \leq$ $+3, \quad 1.1 \leq \tan \beta \leq 50$, and taking into account the experimental constraints previously mentioned. The ensuing explored range for $m_{\chi}$ is from its lower bound up to $250 \mathrm{GeV}$.

Within the present theoretical framework we have evaluated the cross-sections $\sigma_{\text {scalar }}^{(\text {nuclen })}$ and $\sigma_{S D}^{(\text {proton })}$. The results for $\sigma_{\text {scalar }}^{(\text {nucleon })}$, multiplied by the scaling factor $\xi$, are shown in Fig.4. Allowed neutralino configurations stay inside the outer contour line. To provide more information, also the locations of configurations with the representative values: $60 \mathrm{GeV} \leq$ $m_{A} \leq 70 \mathrm{GeV}$ and $\tan \beta=10,50$ are displayed. The thick curve represents the experimental upper bound to $\sigma_{\text {scalar }}^{\text {(nucleon })}$, deduced by using the set I of Table I; thus this curve is just a part of the one in Fig.1 and is due to the new data from the DAMA/NaI experiment. This figure shows that a large number of neutralino configurations are above the current experimental bound. It follows that the present experimental sensitivities have reached a level of great significance for the possibility of investigating realistic supersymmetric models. However, this result cannot be used to disallow those configurations which provide a $\xi \cdot \sigma_{\text {scalar }}^{(\text {nuclon })}$ above the experimental limit, because of the uncertainties affecting the astrophysical parameters. If we assign to these parameters their most conservative values, i.e. the values of set II of Table I, we obtain the results displayed in Fig.5. This Figure shows that, even in the very conservative scenario represented by set II, for the first time the boundary of the physical region for neutralinos is reached by direct detection measurements. From our results it turns out that the neutralino configurations currently accessible by direct detection are those pertaining to light Higgs masses: $m_{A} \simeq 60 \mathrm{GeV}$ and large $\tan \beta: \tan \beta \simeq 40-50$. These same configurations are at present under investigation at LEP2 (at $\sqrt{s}=172 \mathrm{GeV})$.

A similar analysis, performed for the spin-dependent case, shows that the theoretical predictions within MSSM for $\sigma_{S D}^{(\text {proton })}$ are still much below the current upper bound of Fig.3 (by more than two orders of magnitude). This is expected, in view of the much reduced strength of spin-dependent effects versus the coherent ones.

A few comments are in order here. Investigations of fundamental particle-physics properties by non-accelerator experiments usually suffer from a number of serious uncertainties. This is the case for the problem under discussion, as we have already recalled above, in connection with the astrophysical and cosmological parameters. For this reason, in our conclusions we have also conservatively taken into account the set II for these parameters. However, it is remarkable that, even in this unfavorable scenario, the present sensitivity of experiments for direct WIMP search turns out to be at a level of great physical interest for 
supersymmetric relic particles. Significant improvements in the determination of fundamental cosmological parameters (such as the average matter density and the Hubble constant) are expected in the near future; these will allow to draw stronger conclusions from investigations similar to the one presented here. It is also to be remarked that further substantial improvements in the sensitivities of experiments searching for WIMPs are expected, thus the perspectives for this kind of experimental investigation are very encouraging.

\section{CONCLUSIONS}

Using the results of an experiment for direct detection of Weakly Interacting Massive Particle (WIMP), which employs a large-mass, low-background $\mathrm{NaI}(\mathrm{Tl})$ detector at the Gran Sasso Laboratory, DAMA/NaI [1], and new nuclear-physics evaluations of spin-dependent structure functions [2, 3], we have shown that these new experimental data provide the most stringent upper bounds both to scalar and to spin-dependent WIMP-nucleon cross-sections (except for a very narrow range for WIMP mass around $15 \mathrm{GeV}$ ). Applying these results to the most favorite candidate for cold dark matter, the neutralino, we have proved that, for

the first time, data from an experiment of direct search for WIMPs allow the exploration of a region of the supersymmetric parameter space not yet fully investigated at accelerators. This result has been obtained by using for the astrophysical and cosmological parameters a very conservative set of values. We may also conclude that the experiment of Ref. [1] can allow an investigation of modulation effects with significant sensitivity.

\section{Acknowledgments}

We thank Rita Bernabei for very interesting discussions. We also express our thanks to M.Ted Ressell for providing us with unpublished results on spin-dependent nuclear structure functions. 


\section{REFERENCES}

[1] R. Bernabei et al., preprint ROM2F-96/43 (to appear in Phys. Lett. B389 (1996) 757).

[2] M.T. Ressell and D.J. Dean, Proc. of the Int. Workshop on "Identification of Dark Matter", Sheffield, September 1996.

[3] M.T. Ressell, private communication.

[4] S. P. Ahlen et al., Phys. Lett. B195 (1987) 603; D. O. Caldwell et al., Phys. Rev. Lett. 61 (1988) 510.

[5] D. Reusser et al., Phys. Lett. B255 (1991) 143.

[6] H. Ejiri, K. Fushimi and H. Ohsumi, Phys. Lett. B 317 (1993) 14

[7] K. Fushimi et al., Phys. Rev. C 47 (1993) R425.

[8] M. Beck (Heidelberg-Moscow Collaboration), Nucl. Phys. B (Proc. Suppl.) 35 (1994) 150; M. Beck et al., Phys. Lett. B336 (1994) 141.

[9] A.K. Drukier et al., Nucl. Phys. B(Proc. Suppl.) 28A (1992) 293; I. R. Sagdev, A. K. Drukier, D. J. Welsh, A. A. Klimenko, S. B. Osetrov, A. A. Smolnikov, Nucl. Phys. B (Proc. Suppl.) 35 (1994) 175.

[10] E. Garcia et al., Nucl. Phys. B (Proc. Suppl.) 28A (1992) 286; M. L. Sarsa et al., Nucl. Phys. B (Proc. Suppl.) 35 (1994) 154; E. Garcia et al., Proc. "The Dark Side of the Universe", eds. R. Bernabei and C. Tao (World Scientific, Singapore 1994), p. 216.

[11] P.F. Smith et al., Phys. Lett. B379 (1996) 299.

[12] P. Belli et al., Nucl. Phys. B (Proc. Suppl.) 48 (1996) 62; P. Belli et al., Il Nuovo Cim. C19 (1996), 537;

[13] P. Belli et al., Nucl. Phys. B (Proc. Suppl.) 48 (1996) 60; R. Bernabei, Proc. of the Int. Workshop on "Identification of Dark Matter", Sheffield, September 1996;

[14] R. Bernabei et al., Proc. of the Int. Workshop 2nd RESCEU, Tokyo, November 1996.

[15] A. Alessandrello et al., Phys. Lett. B384 (1996) 316.

[16] M.L. Sarsa et al., Nucl. Phys. (Proc. Suppl.) B48 (1996) 73; M.L. Sarsa et al., Phys. Lett. B 386 (1996) 458.

[17] H. Ejiri, Proc. of the Int. Workshop on Aspects of Dark Matter in Astro- and Particle Physics, Heidelberg, September 1996; Proc. of the Int. Workshop 2nd RESCEU, Tokyo, November 1996.

[18] K. Freese, J. Frieman and A. Gould, Phys. Rev. D37 (1988) 3388

[19] F. J. Kerr, D. Lynden-Bell, Mon. Not. R. Astr. Soc. 221 (1986) 1023.

[20] P.J.T. Leonard and S. Tremaine, Ap.J. 353 (1990) 486.

[21] E.I. Gates, G. Gyuk and M. Turner, Ap. J. 449 (1995) L123

[22] R.A. Flores, Phys. Lett. B215 (1988) 73.

[23] T.K. Gaisser, G. Steigman and S. Tilav, Phys. Rev. D34 (1986) 2206.

[24] M.W. Goodman and E. Witten, Phys. Rev. D31 (1985) 3059.

[25] R. Barbieri, M. Frigeni and G.F. Giudice, Nucl. Phys. B 313 (1989) 725

[26] K. Griest, Phys. Rev. D 38 (1988) 2357; Phys. Rev. Lett. 61 (1988) 666.

[27] R.H. Helm, Phys. Rev. 104 (1956) 1466

[28] S. Scopel, Proc. of the Int. Workshop on Aspects of Dark Matter in Astro- and Particle Physics, Heidelberg, September 1996.

[29] ALEPH Collaboration, preprint CERN-PPE/96-083, July 2, 1996 
[30] J. Ellis, T. Falk, K.A. Olive and M. Schmitt, Proc. of the Int. Workshop on "Identification of Dark Matter", Sheffield, September 1996, hep-ph/9610410.

[31] J. Engel, S. Pittel and P. Vogel, Int. J. Mod. Phys. E 1 (1992) 1

[32] G. Jungman, M. Kamionkowski and K. Griest, Phys. Rep. 267 (1996) 195 and references quoted therein.

[33] M.T. Ressell, M.B. Aufderheide, S.D. Bloom, K. Griest, G.J. Mathews and D.A. Resler, Phys. Rev. D48 (1993) 5519.

[34] J. Ellis and R.A. Flores, Phys. Lett. B 263 (1991) 259.

[35] F. Iachello, L.M. Krauss and G. Maino, Phys. Lett. B 254 (1991) 220.

[36] V.I. Dimitrov, J. Engel and S. Pittel, Phys. Rev. D 51 (1995) R291.

[37] J. Ellis and M. Karliner, Phys. Lett. B 341 (1995) 397.

[38] H. P. Nilles, Phys. Rep. 110 (1984) 1; H. E. Haber and G. L. Kane, Phys. Rep. 117 (1985) 75; R. Barbieri Riv. Nuovo Cim. 11 (1988) 1.

[39] A. Bottino, V. de Alfaro, N. Fornengo, G. Mignola and M. Pignone, Astrop. Phys. 2 (1994) 67; A. Bottino, V. de Alfaro, N. Fornengo, G. Mignola and S. Scopel, ibidem 77.

[40] L. Bergström and P. Gondolo, Astrop. Phys. 5 (1996) 263.

[41] A. Bottino, Proc. of the Int. Workshop on "Identification of Dark Matter", Sheffield, September 1996, astro-ph/9611137.

[42] G. Mignola, Proc. of the Int. Workshop on "Identification of Dark Matter", Sheffield, September 1996, astro-ph/9611138.

[43] OPAL Collaboration, CERN Preprint CERN-PPE/96 October 4, 1996; M. Martinez, W. De Boer, M. Pohl, A.T. Watson representing the ALEPH, DELPHI, L3 and OPAL Collaborations, joint seminar at CERN, October 8, 1996.

[44] J. Timmermans (DELPHI Collaboration), DELPHI status report, preprint DELPHI 96-162 GEN 167, November 19, 1996

[45] M.S. Alam et al. (CLEO coll.), Phys. Rev. Lett. 74 (1995) 2885. 


\section{Figure Captions}

Figure 1 - Upper bounds on $\sigma_{\text {scalar }}^{(\text {nucleon })}$. The dot-dashed line denotes the limit obtained by combining data of Ge-detectors; the branch for $m_{\chi} \lesssim 10 \mathrm{GeV}$ is from results of Ref. [10], the one for $10 \mathrm{GeV} \lesssim m_{\chi} \lesssim 20 \mathrm{GeV}$ is from results of Ref. [5] and the one for $m_{\chi} \gtrsim 20 \mathrm{GeV}$ is from data of Ref. [9]. The solid and the long-dashed lines denote the bounds from the results of NaI-detectors: the solid line is from data of Ref. [1], the long-dashed line is from data of Ref. [11]. Dotted and short-dashed lines are from data of a Xe-detector [13] and of a $\mathrm{TeO}_{2}$-detector [15], respectively.

Figure 2 - Structure function $\left[S(q) / a_{p}^{2}\right]_{Z}$, obtained from the results of Refs. [2,3], versus qR. The two lower curves refer to ${ }^{129}$ Xe with the Bonn A (long-dashed line) and with the Nijmegen II (dot-dashed line) nucleon-nucleon potentials. The two upper curves refer to ${ }^{127} \mathrm{I}$ with the Bonn A (short-dashed line) and with the Nijmegen II (solid line) nucleon-nucleon potentials.

Figure 3 - Upper bounds on $\sigma_{S D}^{(\text {proton })}$. The solid and the dashed lines denote the bounds from the results of NaI-detectors: the solid line is from data of Ref. [1], the dashed line is from data of Ref. [11]. The dotted line is from data of a Xe-detector [13 and the dot-dashed line is from Ge-detectors [5,9].10].

Figure $4 \sigma_{\text {scalar }}^{(\text {nucleon })}$, multiplied by the scaling factor $\xi$, versus the neutralino mass. Allowed neutralino configurations stay inside the outer contour line. Configurations with 60 $\mathrm{GeV} \leq m_{A} \leq 70 \mathrm{GeV}$ and $\tan \beta=50(\tan \beta=10)$ stay in the up-left to bottom-right hatched region (in the doubly hatched region). The thick line denotes the upper bound obtained from data of Ref. [1]. The set I of Table I for the astrophysical and cosmological parameters has been used.

Figure 5 The same as in Fig.4 except that set II of Table I for the astrophysical and cosmological parameters has been used. 


$$
\sigma_{\text {scalar }}^{(\text {nucleon) }} \text { (nbarn) }
$$

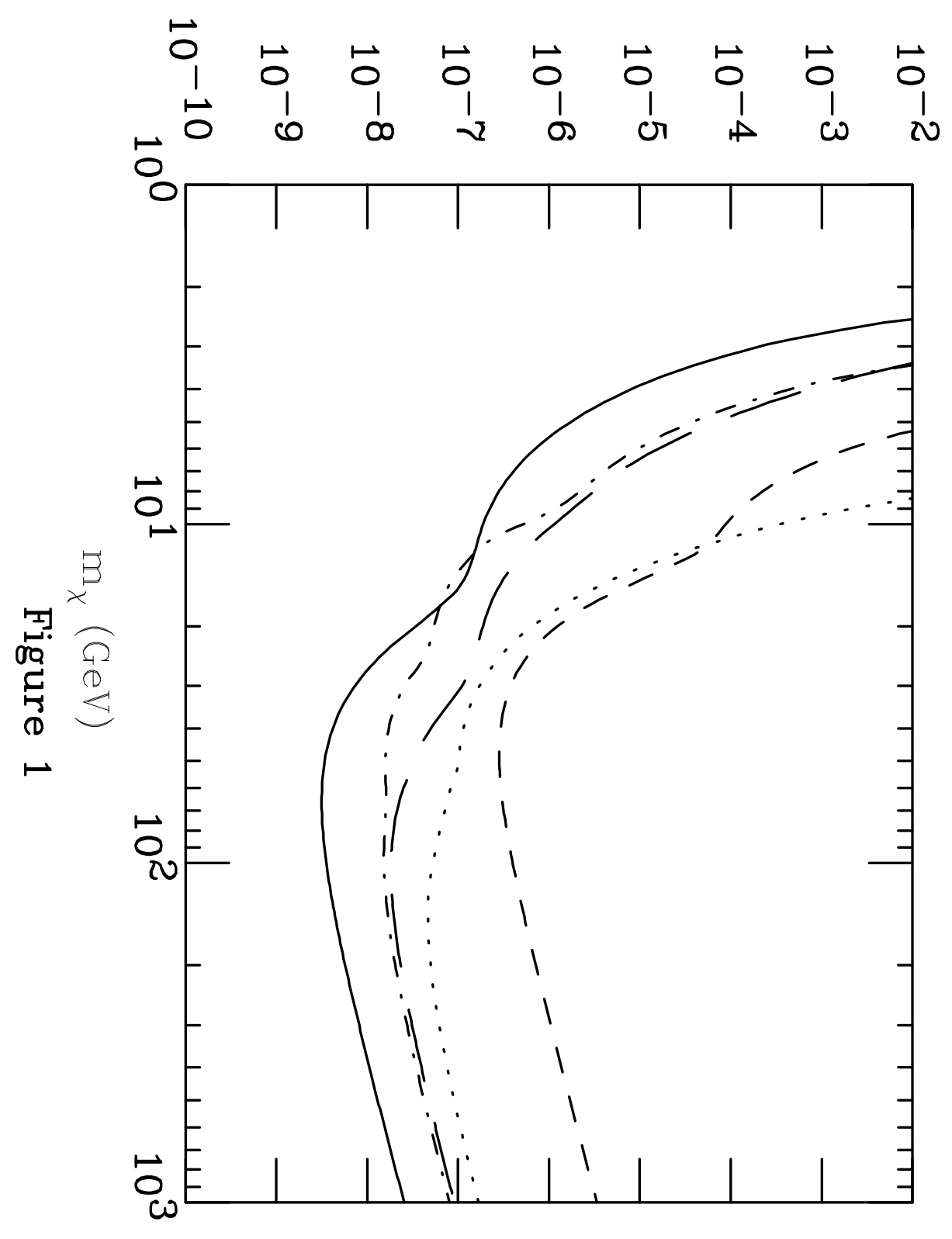




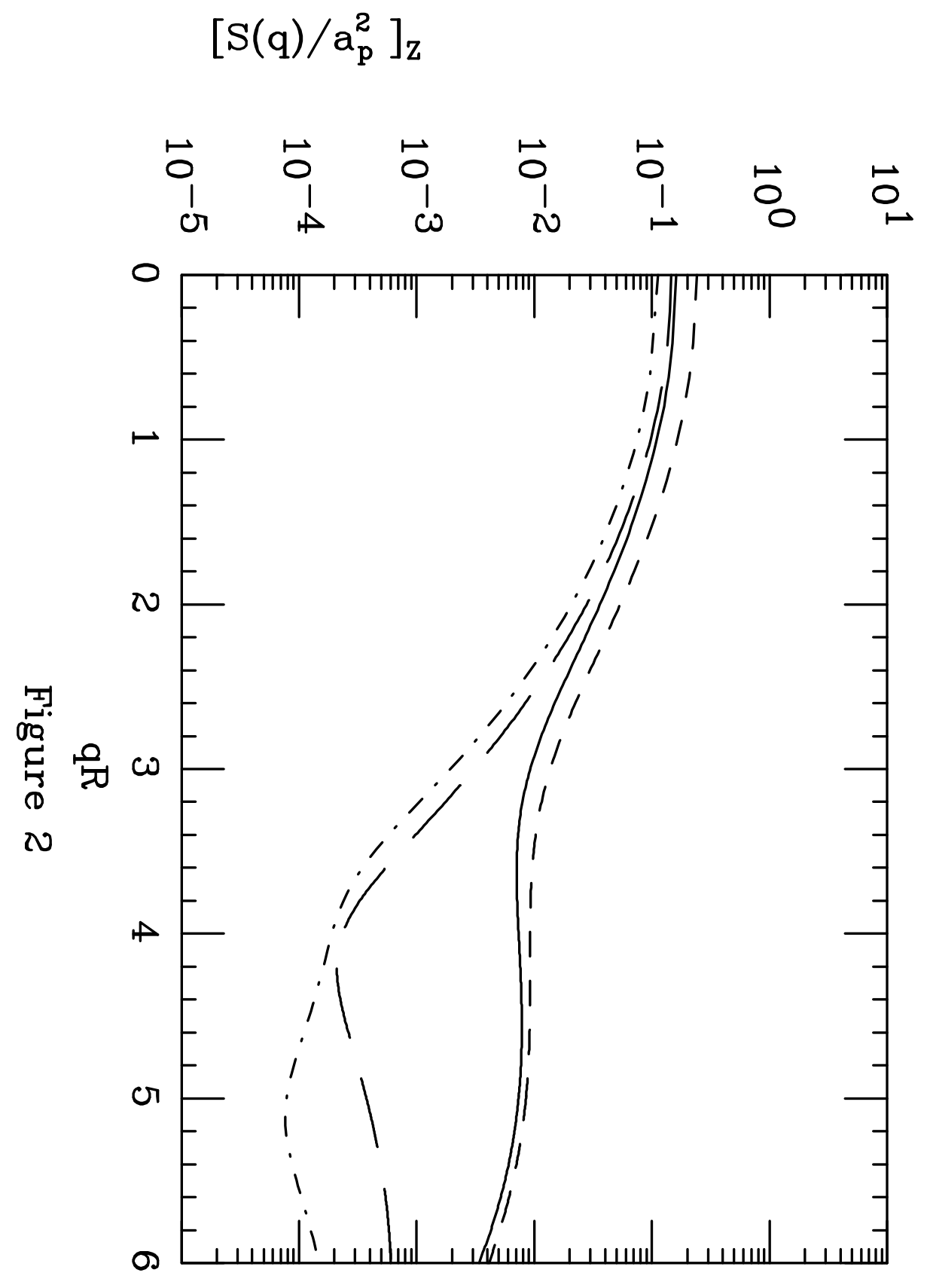




$$
\sigma_{\mathrm{SD}}^{\text {(proton) }} \text { (nbarn) }
$$

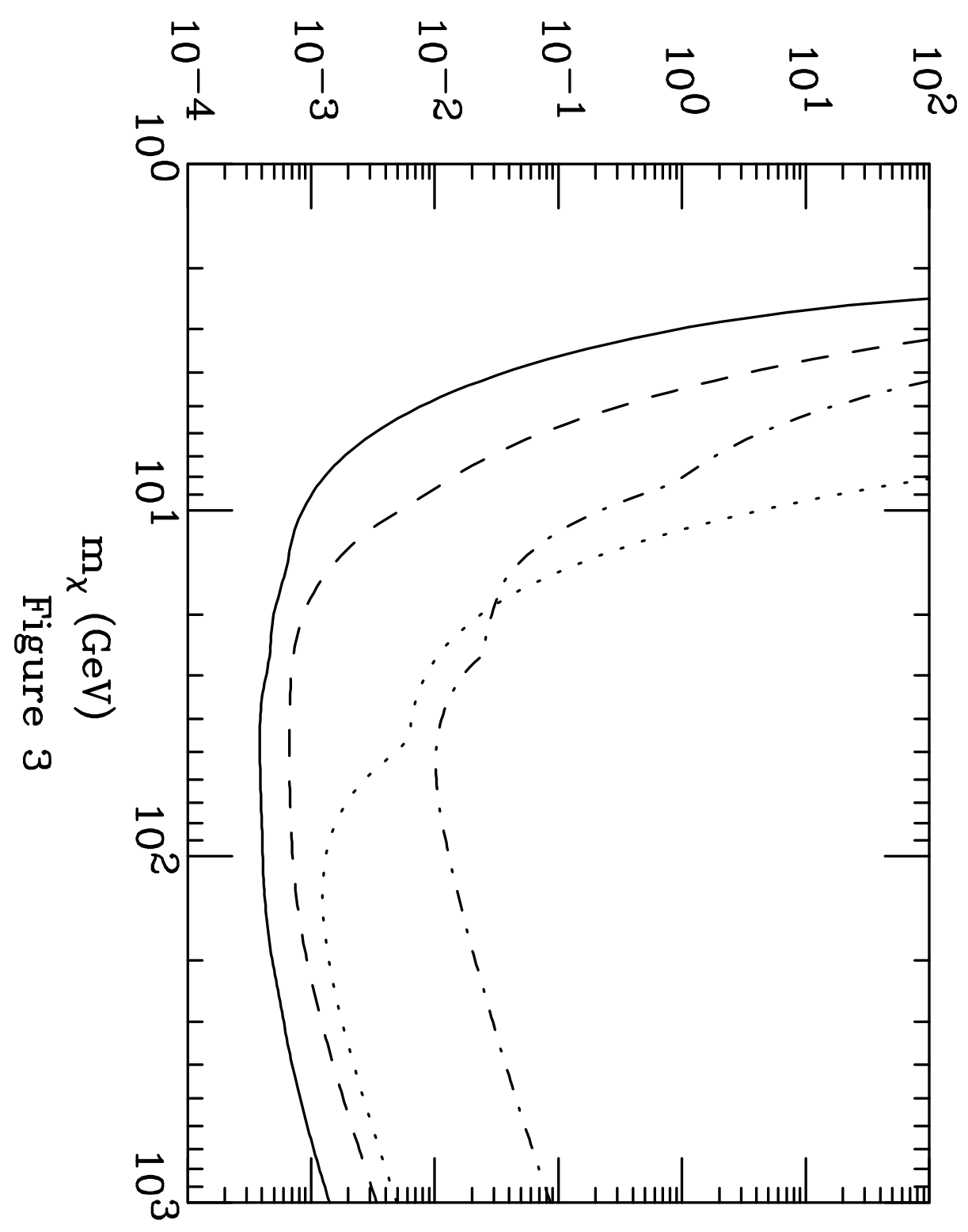




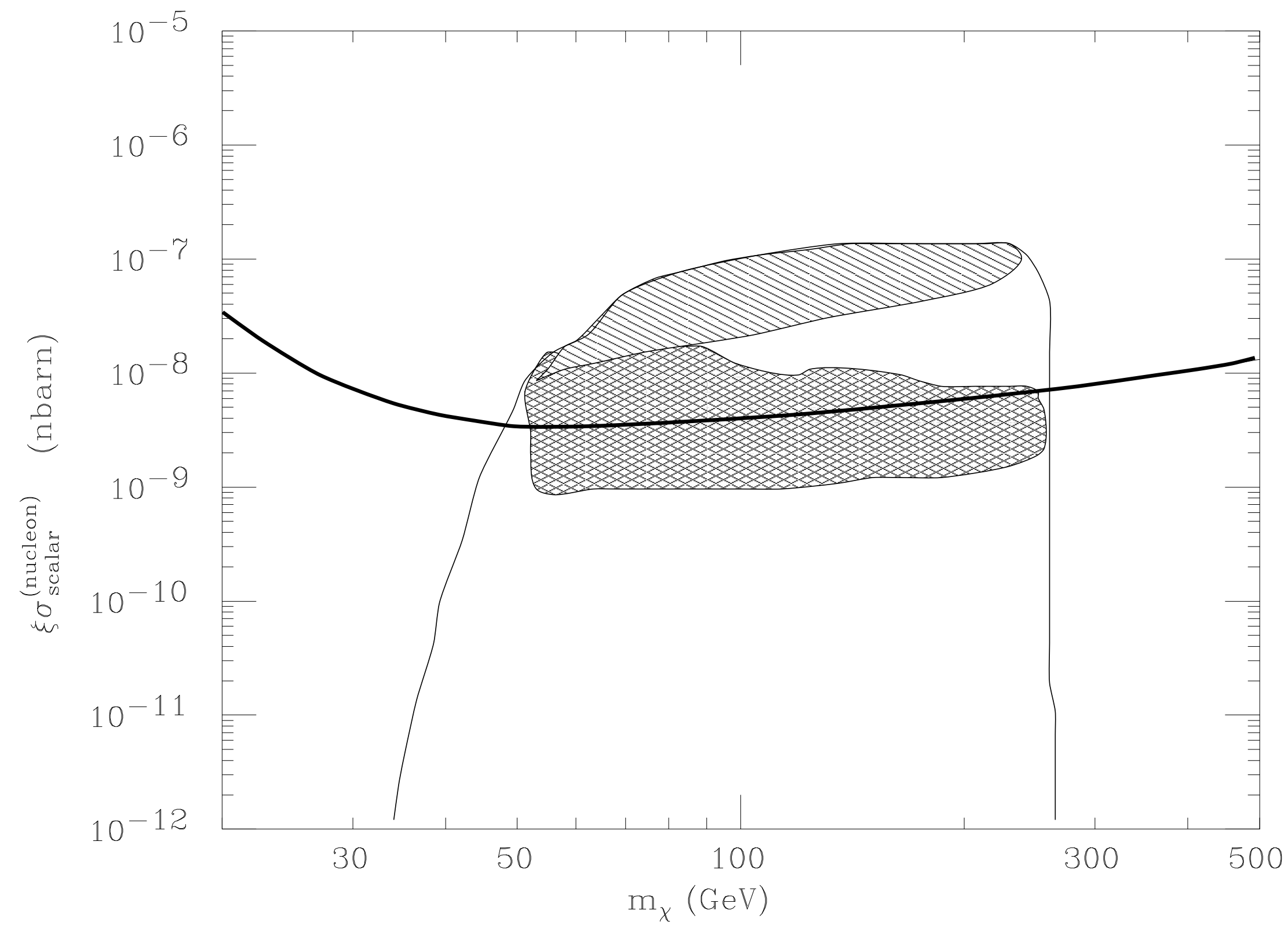

Figure 4 


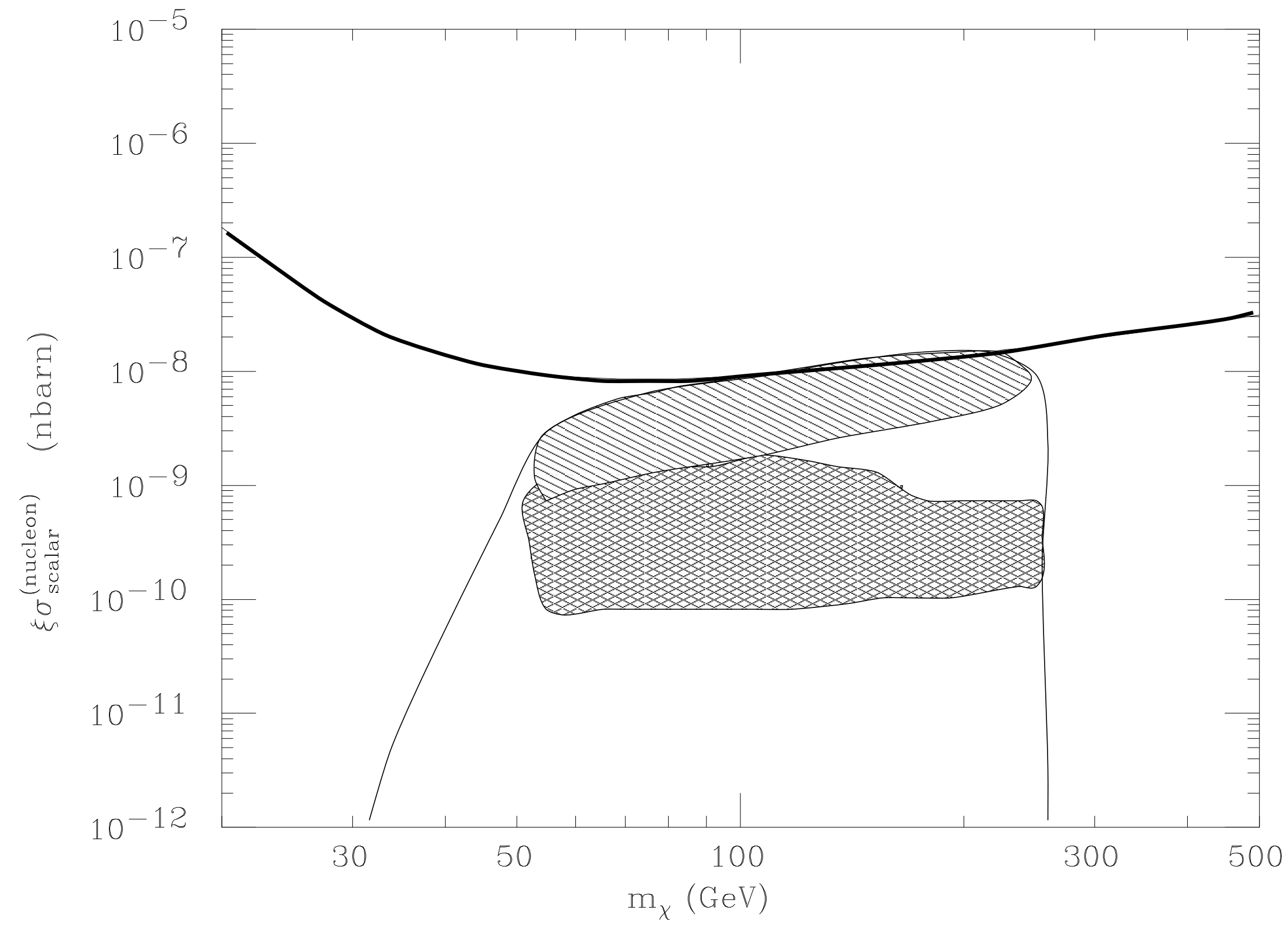

Figure 5 